\title{
THE SCHUR MULTIPLICATOR OF METACYCLIC GROUPS ${ }^{1}$
}

\author{
F. RUDOLF BEYL
}

\begin{abstract}
The Schur multiplicator $\mathrm{H}_{2} \mathrm{G}$ of a (finite) metacyclic group $G$ is computed with the help of the Lyndon spectral sequence. The order of $\mathrm{H}_{2} G$ is a useful invariant of $G$. A metacyclic group with vanishing Schur multiplicator can be presented with two generators and two relators. A simple description of the totality of these groups is given and all such $p$-groups are classified.
\end{abstract}

1. Introduction. All groups in this note are finite. The group $G$ is called metacyclic if it has a cyclic normal subgroup which yields a cyclic factor group. If $M$ and $N$ are natural numbers, and $r$ and $\lambda$ integers, and $r^{N} \equiv 1 \bmod M$, then we introduce

$$
\begin{aligned}
& G(M, N, r, \lambda) \\
& \quad=\left(a, b: a^{M}=1, b \cdot a \cdot b^{-1}=a^{r}, b^{N}=a^{M \lambda /(M, r-1)}\right) .
\end{aligned}
$$

Obviously, $G(M, N, r, \lambda)$ is supported by the metacyclic extension

$$
e(M, N, r, \lambda):{ }^{r} \mathbf{Z}_{M I} \Delta^{*} \longrightarrow G(M, N, r, \lambda) \stackrel{\pi}{\longrightarrow} C_{N},
$$

where $C_{N}$ is the cyclic group of order $N$ and $\mathbf{Z}_{M}$ the additive cyclic group of order $M$, and the distinguished generator $c$ of $C_{N}$ operates on $\mathbf{Z}_{M}$ by multiplication by $r$. (We indicate this by the superscript.) Moreover, $\pi$ maps $b$ to $c$ and $a$ to 1 and $\kappa$ embeds $\mathbf{Z}_{M}$ as the cyclic subgroup of $G$ generated by $a$. It is well known [6, III §7] that all metacyclic groups can be presented as in (1). Our main result is that the Schur multiplicator $\mathrm{H}_{2} G(M, N, r, \lambda)$ is the cyclic group of order $(\lambda, h(M, N, r))$ where

$$
h(M, N, r)=M^{-1} \cdot(M, r-1) \cdot\left(M, 1+r+\cdots+r^{N-1}\right)
$$

Received by the editors January 29, 1973.

AMS (MOS) subject classifications (1970). Primary 18H10, 20F05, $20 \mathrm{~J} 05$.

Key words and phrases. Metacyclic group, Schur multiplicator, Schur group, deficiency, Lyndon spectral sequence.

1 The contents of this paper were included in the author's thesis, The classification of metacyclic p-groups, and other applications of homological algebra to group theory, presented to the Faculty of the Graduate School of Cornell University in partial fulfilment of the requirements for the Ph.D. degree. 
is the order of $H^{2}\left(C_{N} ;{ }_{r} \mathbf{Z}_{M}\right)$. We have proved in [1, $\S 4$ that $G(M, N, r, \lambda)$ $\approx G\left(M, N, r, \lambda^{\prime}\right)$ where $\lambda^{\prime}=(\lambda, h(M, N, r))$. Thus we may assume $\lambda \mid h(M, N, r)$ and then $\lambda$ is an invariant of $G(M, N, r, \lambda)$, viz. the order of $\mathrm{H}_{2} G(M, N, r, \lambda)$. We call $G$ a Schur group if $\mathrm{H}_{2} G=0$. Hence the metacyclic Schur groups are precisely all

$$
\begin{aligned}
& G(M, N, r, 1) \\
& \quad=\left(a, b: a^{M}=1, b a b^{-1}=a^{r}, b^{N}=a^{M /(M \cdot r-1)}\right) .
\end{aligned}
$$

If one searches for Schur groups, it is particularly interesting to know all Schur $p$-groups. The metacyclic ones among these are classified in $\S 5$. Now the Schur multiplicator is related to presentations of $G$ as follows. If $P$ is a presentation of the group $G$ with $n$ generators and $m$ relators, then $(n-m)$ is called the deficiency of $P$. The deficiency of $G, \operatorname{def}(G)$, is the maximum of the deficiencies of all finite presentations of $G$. Then

$$
\operatorname{def}(G) \leqq-d\left(H_{2} G\right)
$$

where $d\left(\mathrm{H}_{2} \mathrm{G}\right)$ is the minimum mumber of generators for the group $\mathrm{H}_{2} G$, cf. Epstein [2, Lemma 1.2]. Hence, if $G$ has a presentation with as many relators as generators, then it is a Schur group. Can the Schur groups (3) be presented with two generators and only two relators? Yes, as shown in $\S 4$. This makes for an independent proof that the groups (3) are Schur groups. Epstein [2] calls a group efficient if equality holds in (4). It is immediate from the results mentioned that all metacyclic groups are efficient.

We thank William Scott in Salt Lake City, Utah, for informing us that Wamsley [5] already computed the Schur multiplicator of metacyclic groups and also proved the efficiency of metacyclic groups. Wamsley's methods are very computational. We compare the results below.

2. Proposition. A metacyclic extension

$$
e:{ }^{r} \mathbf{Z}_{M} \Delta G \stackrel{\pi}{\longrightarrow} C_{N}
$$

determines a long exact sequence

$$
H_{3} G \stackrel{H_{3 \pi}}{\longrightarrow} Z_{N} \stackrel{d^{2}}{\longrightarrow} H_{1}\left(C_{N} ;{ }^{r} Z_{M}\right) \longrightarrow H_{2} G
$$

which is natural with respect to maps of metacyclic extensions.

Proof. Let $(E, d)$ be the Lyndon spectral sequence of the extension $e$ 
for integral homology. The relevant part of the $E^{2}$-chart is

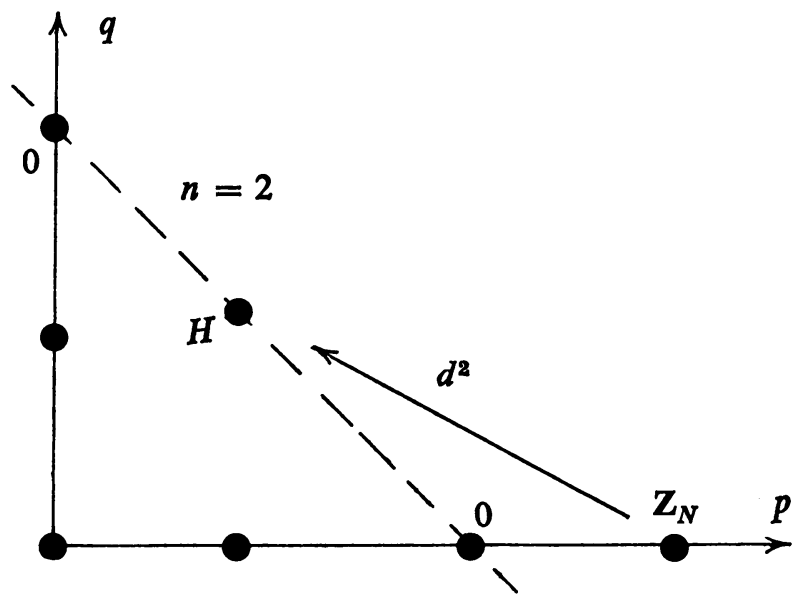

where $H$ stands for $H_{1}\left(C_{N} ;{ }^{r} Z_{M}\right)$. This gives immediately $E_{2,0}^{\infty}=E_{0,2}^{\infty}=0$ and $E_{3,0}^{\infty}=\operatorname{Ker} d^{2}$ and $E_{1,1}^{\infty}=H / \operatorname{Im} d^{2}$. We also know that the composite $H_{3} G \rightarrow E_{3,0}^{\infty} \succ \rightarrow \mathrm{Z}_{N}$ is just $H_{3} \pi$. Since $E$ converges to $H_{*} G$, we obtain the exactness of (5). This sequence is natural in $e$, since so is the Lyndon spectral sequence.

3. TheOREM. Let $e(M, N, r, \lambda)$ be a metacyclic extension as in (1). Then $\mathrm{H}_{2} G(M, N, r, \lambda)$ is the cyclic group of order $(h(M, N, r), \lambda)$ and the image of $\mathrm{H}_{3} \pi: \mathrm{H}_{3} \mathrm{G} \rightarrow \mathrm{H}_{3} \mathrm{C}_{\mathrm{N}}$ is the cyclic group of order

$$
N \cdot(h(M, N, r), \lambda) / h(M, N, r) .
$$

Proof. Using the result of [1] quoted in the introduction, we replace $\lambda$ by $(\lambda, h(M, N, r))$ and call this again $\lambda$. Now $\lambda \mid h(M, N, r)$. Consider the commutative diagram

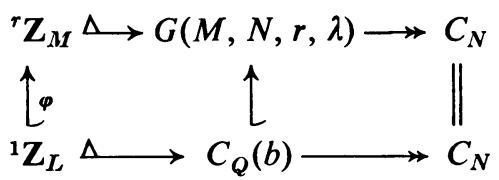

where $L=(M, r-1) / \lambda$ and $Q=L \cdot N$ and $C_{Q}(b)$ is the cyclic group of order $Q$ generated by $b$ in $G(M, N, r, \lambda)$ and ${ }^{1} Z_{L}$ is the additive cyclic group order $L$ generated by $b^{N}=a^{M / L}$. Applying Proposition 2 to this, we obtain a commutative diagram

$$
\begin{aligned}
& H_{3} G \longrightarrow \mathrm{Z}_{N} \stackrel{d^{2}}{\longrightarrow} H \longrightarrow H_{2} G \\
& \| \uparrow_{\Psi=H_{1}\left(C_{N} ; H_{1}(\varphi)\right)} \\
& \mathbf{Z}_{N} \longrightarrow \mathbf{Z}_{(N . L)} \longrightarrow 0
\end{aligned}
$$


with exact rows, where $H=H_{1}\left(C_{N} ;{ }^{r} \mathbf{Z}_{M}\right)$. Since $\mathbf{Z}_{N} \rightarrow \mathbf{Z}_{(N, L)}$ is epic, $\operatorname{Im} d^{2}=\operatorname{Im} \Psi$. We claim Coker $\Psi=Z_{\lambda}$. Assuming this claim, we have $H_{2} G=$ Coker $d^{2}=$ Coker $\Psi=Z_{\lambda}$. We conclude directly from (5) that $N \cdot\left(\# H_{2} G\right)=\left(\# \operatorname{Im}\left(H_{3} \pi\right)\right) \cdot(\# H)$ and that $\operatorname{Im}\left(H_{3} \pi\right)$ is cyclic. Since here the Herbrand quotient is 1 , indeed $\# H=h(M, N, r)$.

Let $r^{*}$ denote the endomorphism of any abelian group which is multiplication by the integer $r$. It is well known that the functor $H_{1}=H_{1}(\cdot, \mathrm{Z})$ is just abelianization. Hence $H_{1}\left(r^{*}\right)=r^{*}$ and $H_{1}(\varphi)=\varphi$. Thus $H_{1}\left({ }^{r} Z_{M}\right)=$ ${ }^{r} \mathbf{Z}_{M}$ and $H_{1}\left({ }^{1} Z_{L}\right)={ }^{1} Z_{L}$ as $C_{N}$-modules and $H_{1}\left(C_{N} ; H_{1}(\varphi)\right)$ is just $H_{1}\left(C_{N} ; \varphi\right): \mathbf{Z}_{(N, L)} \rightarrow H_{1}\left(C_{N} ;{ }^{r} \mathbf{Z}_{M}\right)$. If we compute $H_{1}\left(C_{N} ; \cdot\right)$ by the standard formula [3, VI (7.7)], then all of $Z_{L}$ consists of cycles whereas the cycle group for $H_{1}\left(C_{N} ;{ }^{r} Z_{M}\right)$ is generated by $a_{0}=(M /(M, r-1)) a$. The inclusion $\varphi$ maps the generator $(M / L) a=(M \lambda /(M, r-1)) a$ of $\mathbf{Z}_{L}$ onto $\lambda a_{0}$. Since now $\lambda \mid h(M, N, r)$, this implies Coker $\Psi=\mathbf{Z}_{\lambda}$ as claimed.

4. TheORem. A metacyclic group can be presented with two generators and two relators if and only if it is a Schur group.

Proof. The "only if" part is given by the introduction. For the converse, let the Schur group $G=G(M, N, r, 1)$ be presented as in (3). Clearly, there are integers $s_{1}$ and $k_{1}$ with $(M, r-1)=s_{1}(r-1)+k_{1} M$. Now $s_{1}$ is prime to $V=M /(M, r-1)$. Let $d$ be the greatest factor of $M$ prime to $s_{1}$ and $s=s_{1}+d V$. We need only the following properties of $s$ :

$$
(M, r-1) \equiv s(r-1) \bmod M \text { and }(M, s)=1 .
$$

Since $\left[b, a^{s}\right]={ }_{\mathrm{def}} b \cdot a^{s} \cdot b^{-1} \cdot a^{-s}=a^{(r-1) s}=a^{(M, r-1)}$ in $G(M, N, r, 1)$, the following relations (b) and (c) clearly hold in $G(M, N, r, 1)$ :

$$
\begin{gathered}
b^{N}=a^{V} \quad \text { with } V=M /(M, r-1), \\
{\left[b, a^{s}\right]=a^{(M . r-1)} .}
\end{gathered}
$$

We claim that (b) and (c) generate the group of relators. We conclude from (b) that $a^{V}$ commutes with $b$, and from (c) that $\left[b, a^{s}\right]$ commutes with $a$. From this we obtain

$$
a^{s V}=b \cdot a^{s V} \cdot b^{-1}=\left(b \cdot a^{s} \cdot b^{-1}\right)^{V}=\left(\left[b, a^{s}\right] \cdot a^{s}\right)^{V}=\left[b, a^{s}\right]^{V} \cdot a^{s V}
$$

or $\left[b, a^{s}\right]^{V}=1$. This and (b) and (c) give

$$
a^{M}=1 \text {. }
$$

There is an integer $t$ with

$$
s ! \cdot t \equiv 1 \bmod M .
$$


Now (d) and (e) and (a) give $[b, a]=\left[b, a^{s t}\right]=\left(\left[b, a^{s}\right] \cdot a^{s}\right)^{t} \cdot a^{-s t}=\left[b, a^{s}\right]^{t}=$ $a^{t(M, r-1)}=a^{r-1}$ which is the last of the original relations.

5. Classification of metacyclic noncyclic Schur $p$-groups. The isomorphism types of metacyclic $p$-groups have been determined in $[1, \S 6]$. When we select those classes of noncyclic groups which have a representative with $\lambda=1$, we obtain the following list.

$$
\begin{array}{rll}
\text { I } & G\left(p^{m}, p^{n}, p^{m-n}+1,0\right) & p \text { odd, } m>n \geqq 1 ; \\
\text { II } & G\left(p^{m}, p^{n}, p^{k}+1,1\right) & p \text { odd, } n \geqq 3,2 n-2>m>2, \\
& & \frac{m}{2}>k \geqq \max (1, m-n+1) ; \\
\text { III } \quad G\left(2^{m}, 2^{n}, 2^{m-n}+1,0\right) & m-2 \geqq n \geqq 1 ; \\
\text { IV } \quad G\left(2^{m}, 2^{n}, 2^{m-n}-1,0\right) & m-2 \geqq n \geqq 1 ; \\
\text { V } \quad G\left(2^{m}, 2^{n}, 2^{k}+1,1\right) & n \geqq 4,2 n-2>m>4, \\
& & \frac{m}{2}>k \geqq \max (2, m-n+1) ; \\
\text { VI } G\left(2^{m}, 2,-1,1\right) & m \geqq 2 ; \\
\text { VII } G\left(2^{m}, 2^{n}, 2^{k}-1,1\right) & n \geqq 2, m \geqq 3, \\
& & m>k \geqq \max (2, m-n+1) .
\end{array}
$$

The groups listed are pairwise nonisomorphic. The groups I, III, and IV are split metacyclic, the others cannot be supported by a split metacyclic extension. One has $G\left(2^{m}, 2^{n}, 2^{m-1}-1,1\right) \approx G\left(2^{m}, 2^{n},-1,1\right)$ in case VII. The groups VI are the generalized quaternion groups. Schur [4, pp. 107108] himself knew already that the following are Schur groups: IV for $n=1, \mathrm{I}, \mathrm{III}$, and VI.

6. Comparison with Wamsley [5]. In [5, Lemma 1] the order $x$ of the Schur multiplicator is a GCD of six numbers. If one translates his notation into ours by starting with $a \mapsto a^{-1}$ and $b \mapsto b^{-1}$ and uses standard properties of GCD's, one can obtain

and then

$$
x=\operatorname{GCD}\left\{\lambda,(M, r-1), \frac{(M, r-1)}{M} \cdot \frac{r^{N}-1}{r-1}\right\}
$$

$$
x=\operatorname{GCD}\left\{\hat{\lambda}, M^{-1} \cdot(M, r-1) \cdot\left(M, 1+r+\cdots+r^{N-1}\right)\right\} .
$$

Because of (2), this agrees with our Theorem 3. 
Finally some remarks on [5, Lemma 2] which corresponds to our Theorem 4. Steps (i), (ii), (iii), and (iv) serve to change $r$ and $\lambda$ as to satisfy $(M \lambda /(M, r-1)) \mid M$ and $((r-1) /(M, r-1), M)=1$. The first condition is automatic in our approach from $h(M, N, r) \mid(M, r-1)$, the second superfluous. In case the parameters satisfy both conditions, Wamsley's presentation of the metacyclic Schur group $G$ coincides with ours modulo arbitrary choices.

\section{REFERENCES}

1. F. R. Beyl, The classification of metacyclic p-groups (to appear).

2. D. B. A. Epstein, Finite presentations of groups and 3-manifolds, Quart. J. Math. Oxford Ser. (2) 12 (1961), 205-212. MR 26 \#1867.

3. P. J. Hilton and U. Stammbach, $A$ course in homological algebra, Graduate Texts in Math. 4, Springer-Verlag, New York and Berlin, 1971.

4. I. Schur, Untersuchungen über die Darstellungen der endlichen Gruppen durch gebrochene lineare Substitutionen, J. Reine Angew. Math. 132 (1907), 85-137.

5. J. W. Wamsley, The deficiency of metacyclic groups, Proc. Amer. Math. Soc. 24 (1970), 724-726. MR 41 \#3576.

6. H. Zassenhaus, Lehrbuch der Gruppentheorie, Teubner, Leipzig, 1937; English transl., Chelsea, New York, 1949. MR 11, 77.

Mathematisches Institut, 69 Heidelberg 1, Im Neuenheimer Feld 9, West GERMANY 